\title{
68. A Possible Explanation of the Phenomenon Analogous to "Beats," which often Occurs in Rapid Periodic Variations of Terrestrial Magnetism.
}

\author{
By Masito NaKano. \\ (Comm. by T. OKaDA, M.I.A., June 12, 1933.)
}

I. It has long been known that the records of the terrestrial magnetic elements obtained by the magnetograph of high sensibility show as a rule numerous trains of more or less regular waves which are called the "micropulsations." This phenomenon seems to have been studied first by Balfour Stewart who, in 1861, found periods of 30 seconds. From 1911 to 1914 Prof. T. Terada observed the micropulsations at Misaki in this country, and on his statistical investigation with a huge amount of data, the periods ranging from about 20 seconds to several minutes were obtained. He pointed out, moreover, that in this kind of terrestrial magnetic variations there occur sometimes a characteristic train of micropulsations showing a feature like that of " beats" in acoustics. Fig. 1 shows such an example. From this figure we see that fairly regular micropulsations with a period of about 2 minutes take place, while the amplitude increases and decreases alternately with a period of about 25 minutes. Thus, it seems to be adequate to suppose that this kind of micropulsations is not an oscillating phenomenon with a single period, but a motion composed of two oscillations with nearly equal periods, $T_{1}$ and $T_{2}$ say. If we express the amplitude $s$ of the micropulsations in the form

$$
s=A_{1} \cos \left(n_{1} t+\varepsilon_{1}\right)+A_{2} \cos \left(n_{2} t+\varepsilon_{2}\right),
$$

where $n_{1}=2 \pi / T_{1}, n_{2}=2 \pi / T_{2}$, then the period of micropulsations $\theta$ and that of beats $\theta$ are expressed as follows:

$$
\theta=4 \pi /\left(n_{1}+n_{2}\right), \quad \theta=4 \pi /\left(n_{2}-n_{1}\right),
$$

$n_{2}$ being assumed to be greater than $n_{1}$. From this we have

$$
n_{1}=2 \pi\left(\frac{1}{\theta}-\frac{1}{\theta}\right), \quad n_{2}=2 \pi\left(\frac{1}{\theta}+\frac{1}{\theta}\right),
$$

accordingly $\quad T_{1}=\frac{\theta \cdot \theta}{\theta+\theta}, \quad T_{2}=\frac{\theta \cdot \theta}{\theta+\theta}$.

In the last expressions, since $\theta$ and $\theta$ are known from the records of observation, the component periods $T_{1}$ and $T_{2}$ can be determined. For 
Fig. 1. North-South-Component. April 3-4, 1913

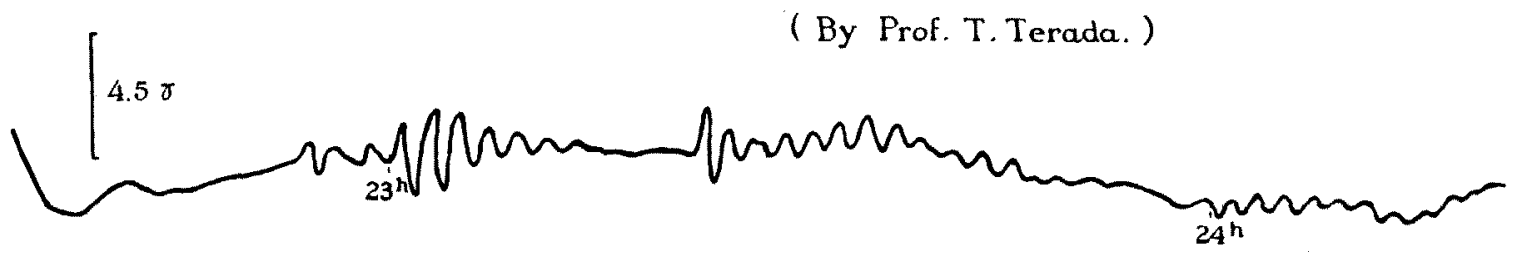

Fig. 2 .

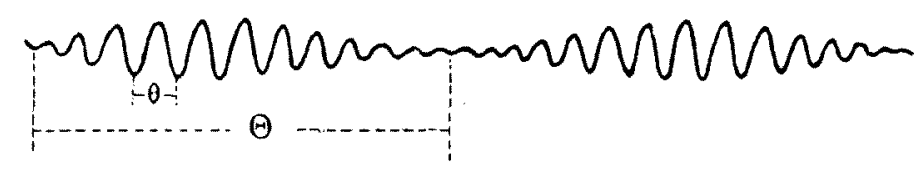

example, the above record obtained by Prof. T. Terada gives $T_{1}=2,2 \mathrm{~min} ., T_{2}=1,9 \mathrm{~min}$.

II. Then, why do such micropulsations occur? In the following I shall propose a provisory explanation for this phenomenon. From the investigations on the transmission of radio waves it has been known that there exists a highly ionized layer in the upper atmosphere, which is called Kennelly-Heaviside layer, and according to the recent authorities in this line there are two such layers, i.e. $E$ - and $F$-layers which are said to be at the heights of about $100 \mathrm{~km}$. and $200 \mathrm{~km}$. respectively from the earth's surface. I think that the existence of the two ionized layers may have a close connection to the micropulsations resembling to "beats." At present the mode of oscillation of these ionized layers is not only entirely obscure, but also even the existence of it is not yet assured. Nevertheless, we may be justified, to some extent, in supposing that these ionized layers oscillate with considerable amplitudes more or less vertically, judging from the observational fact that the heights of the ionized layers in daytime differ from those in night. If this be actually the case, it seems to be very probable that some limited portions of the two ionized layers oscillate with their own natural periods, being accompanied by these larger diurnal oscillations. H. Lamb and the late Prof. S. Sano proved that, in the atmosphere bounded at the lower extrimity by the earth's surface and left free at the upper boundary, there may occur a vertical oscillation having a definite natural period. According to Prof. S. Sano this natural period is approximately given by

$$
T=4 \pi c / r g \text {, }
$$

where $c$ is the velocity of sound, $g$ the acceleration due to gravity, and 
$r$ the ratio of the specific heats, or $c_{p} / c_{v}$. In the atmosphere there can occur various higher-harmonics as well as the fundamental oscillation, as in the case of the vibration of the air column in a pipe closed at one end. The above expression gives the period of the fundamental oscillation, and the periods of the harmonics must be of course less than this. Moreover, we know, as the common property of the oscillating bodies, that what mode of oscillation occurs depends on what portion of the body the external force is applied to. Now, the two ionized layers, $E$ and $F$, may be treated as two charged plates, whose charges are always changing on account of the influence of the various kinds of the solar radiation. Thus, if the two ionized layers be once subjected to disturbances, the atmosphere will oscillate for some time, the loops of vertical oscillation being located nearly in those layers.

Thus, since the change of the place of application of the external force brings about different modes of vibration, the mode of vibration of the upper atmosphere excited in case when the external force is applied to $E$-layer must be different from that which excited in case when the force is applied to $F$-layer. In other words, the proper periods of oscillation of the two layers are more or less different. If we assume, after Schuster's opinion on the origin of the atmospheric current, that currents are induced in the ionized layers on account of their vertical oscillation in the earth's magnetic field, and these currents cause the variations of terrestrial magnetism, then the occurence of the micropulsations resembling to "beats" may be explained qualitatively by considering that the two ionized layers oscillate more or less vertically with slightly different periods.

III. Next I shall calculate the approximate value of the period corresponding to the fundamental oscillation of the atmosphere, given by $T=4 \pi c / r g$.

(A) As a rough estimation, if we put

$$
\begin{aligned}
& g=980 \mathrm{~cm} . / \mathrm{sec}^{2} \quad \text { (the value of } g \text { on the earth's surface.) } \\
& r=1,41 \\
& c=330 \mathrm{~m} . / \mathrm{sec} . \quad \text { (velocity of sound at } 0^{\circ} \mathrm{C} \text { ), }
\end{aligned}
$$

then $T=4 \pi c / r g \doteqdot 300 \mathrm{sec}$. or $5 \mathrm{~min}$.

(B) The temperature in the upper atmosphere is still obscure, but if we assume the value obtained by Lindemann and Dobson, from the investigation on the luminescence of meteors, viz. about $20^{\circ} \mathrm{C}$ to $30^{\circ} \mathrm{C}$, and put 


$$
\begin{aligned}
g & =980 \mathrm{~cm} . / \mathrm{sec}^{2} \\
\gamma & =1,41 \\
c & =344 \mathrm{~m} . / \mathrm{sec} .
\end{aligned}
$$

then $\quad T \fallingdotseq 313$ sec.

(C) On the other hand, according to A. Wegener, W. J. Humphreys and others, the temperature in the upper atmosphere is about $-50^{\circ} \mathrm{C}$ to $-60^{\circ} \mathrm{C}$. If we take $-50^{\circ} \mathrm{C}$ and put

$$
\begin{aligned}
& g=980 \mathrm{~cm} . / \mathrm{sec}^{2} \\
& \gamma=1,41 \\
& c=300 \mathrm{~m} . / \mathrm{sec} . \text { (velocity of sound at }-50^{\circ} \mathrm{C} \text { ), }
\end{aligned}
$$

then $T \fallingdotseq 273 \mathrm{sec}$.

The values of $T$ above calculated are all in good accordance with the greater ones of the actual periods of micropulsations ranging from 20 sec. to 300 sec., which were obtained by Prof. T. Terada from his statistical investigations, and if we consider that, in the oscillation of the atmosphere, there may occur various kinds of harmonics as well as the fundamental oscillation above considered, we are able to explain for shorter periods. 